\title{
An Introduction of Small-scale Intelligent Manufacturing System
}

\author{
Regarding its design objectives and relevant tools and technologies \\ Taoying Huang, Wei Deng Solvang and Hao Yu \\ Department of Industrial Engineering \\ Faculty of Engineering Science and Technology \\ UiT - The Arctic University of Norway \\ Narvik, Norway \\ E-mail: huang.taoying@outlook.com, wei.d.solvang@uit.no, hao.yu@uit.no
}

\begin{abstract}
Manufacturing companies in Northern Peripheral and Arctic region are predominately small and medium-sized and face considerable challenges like geographical isolation and a lack of benefits offered by industrial clusters. For the ultimate goal of enhancing their competitiveness in a global market, it is imperative for companies to innovate or adopt innovations in order to quickly response to changes in market, meet customer demands, reduce time-to-market and lower cost. A novel concept for small-scale intelligent manufacturing systems (SIMS) is introduced, in which diverse methods and innovative technologies can be applied and integrated. This paper gives an introduction of SIMS, defines its design objectives, and summarizes major relevant tools, techniques and paradigms for the development of SIMS, to generate a facilitative environment for small and medium-scale manufacturing enterprises to embrace new and innovative technologies.
\end{abstract}

Keywords-SMEs; intelligent manufacturing system; small scale production; flexible manufacturing

\section{INTRODUCTION}

The predominately small and medium-scale enterprises (SMEs) in the thinly dispersed regions of northern peripheral and arctic (NPA) area, especially in the manufacturing sector, play an important role and occupy a strategic place in the socio-economic growth. Despite their high contribution to regional economy, SMEs face considerable challenges like inadequate exposure to international environment due to limitations commonly related to firm size, geographical isolation, standards/quality, supply chains/logistics, market opportunities, and technology innovation [1]. In order to realize the full potential of SMEs, it is imperative to enhance their capacity to innovate and strengthen their competitiveness, along with the growing globalization of markets.

Four main types of a company's competence needs during innovation processes are described as: technical/technological knowledge; strategic/business knowledge; market knowledge; and internal organizational skills [2]. While the main issue for SMEs in the service sector is new business strategy - a shift in ways of doing business, the primary problem faced by small and medium-scale manufacturing companies has been access to new technology. The reasons for the low capability of SMEs to utilize and develop new technology can possibly be: poor adaptability to changes, desire to avoid risk, lack of technological information and access to relevant consultancy services, lack of professionalism regarding both management and technical personnel, low levels of R\&D, etc. [1] To enable SMEs to overcome the above problems and become new technology-based firms for establishing their competitive edge in an international market strongly relies on a conducive environment based on the formulation of pertinent policies and programs and with a shift to knowledge economy and a focus on benefiting from $R \& D$ and collaboration.

Fierce competition on a global level and more demanding customer needs are the driving forces behind a rapid pace of innovation and changes in market and technology. The technical/technological innovation is usually in the form of new products with new technical components or through the use of new technology in the production process [2]. While looking into new approaches and essential measures to support small and medium-sized manufacturing companies with new technologies and advanced processes, a novel concept for small-scale intelligent manufacturing systems (SIMS) is introduced, in which diverse methods and innovative techniques can be applied and integration at different levels (among enterprises, systems, components, methods and technologies, and value networks) is highlighted. Coupling technological innovation with key management and marketing skills such as trend analysis and integrating products into a whole cycle of service delivery will build up the competitive advantage and improve the value of manufacturing companies. Overall, the purpose of developing SIMS is to create a highly integrated, automated and efficient manufacturing environment for SMEs.

The aim of this paper is to define small-scale intelligent manufacturing systems for SMEs through identifying its design objectives and summarize the tools, techniques and paradigms that can be applied to SIMS for the goal of enabling small and medium-scale manufacturing enterprises to embrace the next generation of innovation and technology. 


\section{INTELLIGENT MANUFACTURING SYSTEM FOR SMES}

A highly competitive manufacturing company should be able to react to changes rapidly and cost-effectively [3]. That is to say, an efficient manufacturing system should be designed and implemented to improve the company's performance in terms of quick response to customer demands, reducing time-to-market and lowering costs for production and product development.

In order to achieve the above goals, manufacturing systems in future are required to be more accurate, agile, interoperable, reconfigurable, and generally intelligent through continuous innovation [4]. Based on these targets, a design objective tree for intelligent manufacturing systems (IMS) for SMEs is created, shown in Fig. 1.

\section{A. Objectives}

1) Reliable: The whole manufacturing system should be reliable above all. It can be divided into two aspects.

a) Accurate: Minimized operating errors occur in the manufacturing process in an intelligent system.

b) Safe and robust: Device facilities in the system are capable of preventing theft, explosion and injury to either the device itself or others. In the growing digital world, theft no longer refers to only the shift of the device's geographical location, it can also refer to the loss of digital data that are stored in a device.

2) Agile: Quick and effective response to customer needs and rapid adaptation to unpredictable changes in market or in regulatory requirements is expected. Cooperation between the company and its suppliers, partners and customers should be in a quick-response manner. In a manufacturing system, it can be backed up and realized by real-time scheduling, ordering, tracking, control, etc.

3) Flexible: The system can quickly adjust the system structure, manufacturing processes, material flows and logistical functions in order to improve the capacity and functionality of the entire system with a product family.

4) Reconfigurable: The system should be designed at the outset to be reconfigurable to allow rapid changes in machine structures and components and consequently quick adjustment of production capacity and functionality. Key characteristics of a changeable (either reconfigurable or flexible) system are described as follows [3,5]:

a) Modular: All major components are modular, both hardware and software.

b) Integratable: Modules are designed with interfaces and components can be integrated quickly and easily. Human and software and hardware also need to be integrated to work collectively at various stages of manufacturing process. The system can also integrate with future introduction of new technology such that it is upgradable.

c) Customized: Machine and control modules are capable of providing the exact (no more and no less) functions needed for specific production, thereby either reducing cost or meeting customer expectation.

d) Convertible \& scalable: The system allows quick changeover between batches of an existing product and quick adjustment for future product and production. Scalability is the counterpart characteristic of convertibility. In a reconfigurable system, the term convertibility is adopted; and for a flexible system, scalability is adopted.

e) Diagnosable: The system is designed to be able to effectively detect and recover from failures that occur in product quality and production process.

5) Inter-operable: The system consisting of devices with understandable interfaces should be compatible with devices outside the system itself and other present or future systems, with no restricted access and no need to change in the system. Or that the system (i.e. facilities, devices and products) and humans can connect and communicate with each other.

6) Automated: The devices in the system can work by themselves with little or no direct interventions from human or others, and have control over own actions and internal state.

7) User friendly: An intelligent system demands minimal human effort. Easy operation and maintenance by users are desirable, and it should be easy for users to learn to operate facility.

8) Environment friendly: A green manufacturing system aims to save energy, reduce wastes of materials, and produce minimal pollution to the environment.

\section{B. Additional Requirements}

Other objectives can be for example: aesthetic design of components in the system, minimum space occupancy of facilities, movability of plant for the convenience of after-sale service, etc. These are considered either less imperative or less desirable than the above listed objectives.

It should be noted that although the design objectives are clearly classified, some of the objectives might be closely related. For instance, a reconfigurable and flexible system may contribute to agility. Nevertheless, each objective has its own focal point. Definitions of five types of changeability related to hierarchical product and production levels, including reconfigurability, flexibility and agility, can be found in [6], according to which reconfigurability is an operational ability, flexibility is a tactical ability, and agility is a strategic ability. 
This copy is the accepted manuscript by SIMS 2016 .

The published version is available on IEEE Xplore: http://ieeexplore.ieee.org/document/7802896/

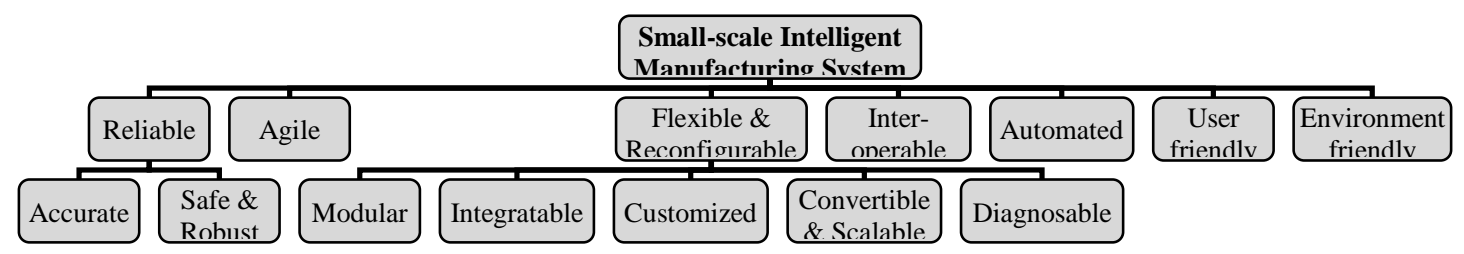

Fig. 1. Design objective tree for intelligent manufacturing system for small and medium-scale manufacturing enterprises.

TABLE I. ToOls, TeChNiques AND TECHNOLOGIES FOR SMALL-SCALE INTELLIGENT MANUFACTURING System

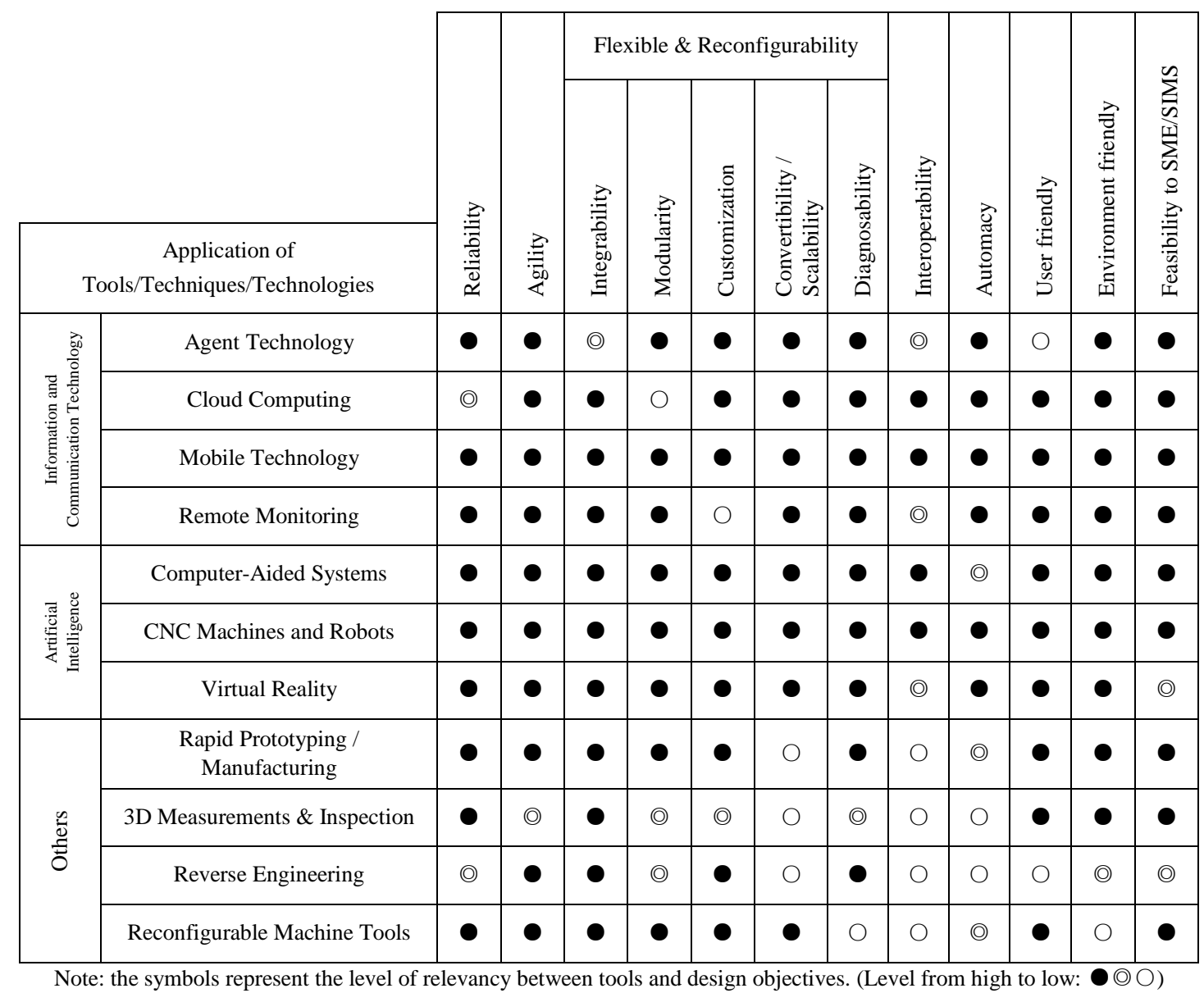

\section{TOOLS AND TECHNOLOGIES FOR SIMS}

The small and medium-sized manufacturing companies mostly conduct low and medium-volume production. The general demand to reduce time-to-market and cost involved in the entire production process has driven companies to turn to innovative technologies. Diverse methods have been continuously developed, and advanced enabling technologies have frequently emerged to be applied to the entire process from conceptualization to prototyping to production.

The new concept of small-scale intelligent manufacturing system is thereby put forward. Intending to improve SMEs' productivity and organizational efficiency, SIMS as the next generation of manufacturing systems for SMEs, can be defined as the integration of the manufacturing enterprise through the adoption of relevant technologies and integrated systems coupled with appropriate managerial philosophies in order to realize optimization through the entire value chain (referring to the definitions of relevant paradigms mentioned hereinafter).

An overview of relevant tools, techniques and technologies for SIMS that can be of great potential for SMEs is presented in Table 1. Particularly, two major categories, information and communication technologies and artificial intelligence techniques, have been developed and used for decades and revolutionizing the development of IMS. Despite that they are in some cases mixed, a clear classification is adopted in this paper. 


\section{A. Information and communication technology}

Development on modern information and communication technology (ICT) has a huge influence on changes of the manufacturing system. An overall integration of manufacturing functions from design to delivery is facilitated through information technology (IT) associated with information management systems. Several pertinent technologies under this category are introduced below for their application to SIMS.

\section{1) Agent Technology}

Agent-based computation is an emerging paradigm of ICT. An agent is a computational system situated in a dynamic environment and is capable of autonomous action in changing circumstances. An agent can make decisions for itself, help in self-recovery and react to real-time perturbations [7].

The common characteristics of SMEs as business range focused, and the trend for SMEs to share business interests, skills and knowledge and collaborate through social networking, strongly call for an open and dynamic environment where efficient and effective responses to changes are expected among more or less independent and cooperative partners. The development in multi-agent systems in the domain of distributed artificial intelligence, addresses the agility, re-configurability, and flexibility requirement, and presents the advantages of decentralization of control over distributed and cooperative structures, robustness, modularity, scalability, diagnosability, customization, autonomy and reusability $[7,8]$. Besides, it is proved by experiments that the implementation of multi-agent technology in an IMS makes the operations much more economical and energy-efficient [9]. For detailed reviews about the application of agent technology in various domains of manufacturing, see in $[7,10,11]$. Introduction of a similar concept of holonic manufacturing system can also found.

Agent technology is vital in the globalization context where the partners are inherently distributed both from geographical and information processing perspectives. However, the open-ended design and implementation of distributed agent-based systems may require high complexity of the system, and the problems caused by agents cannot be solved by less human effort. A multi-agent manufacturing system also requires the inter-operability of a number of agents and the integration of physical automation devices with the software control system for industrial applications, which are still barriers for deployment. An agent-based system also needs reliable distributed infrastructures and is dependent on software performance and quality.

\section{2) Cloud Computing}

Another compelling paradigm that is rapidly changing the landscape of ICT is cloud computing. The commonly used definition of cloud computing is given by the National Institute of Standards and Technology, see in [12]. The computing cloud is an autonomous system and provides users with scalable, flexible and Quality of Service (QoS) guaranteed computing platforms and enables the delivery of on-demand computing resources (applications, services, data, etc.) via a network of remote servers hosted on the Internet through a service-oriented interface. A cloud computing system is thus able to get consistent real-time data, adapt to user needs quickly and eventually gain agility and flexibility in deploying and customizing manufacturing solutions.

Cloud computing technology is changing the way enterprises do their businesses in that dynamically scalable and virtualized resources by supporting new manufacturing business models such as pay-as-you-go/use and business-tobusiness (B2B) solutions for commerce transactions between partners. Cloud-based solutions can also help integrate and streamline business processes by moving a traditional manufacturing process to the cloud networks for improving operational efficiency and creating a more collaborative work environment for SMEs. Having a solution hosted and maintained by experts in secure data centers also means that SMEs can spend more time on innovation and less on IT.

Nevertheless, security, legal and privacy issues are widely acknowledged as being important in cloud computing [13]. Besides, the robustness of a cloud infrastructure is determined by the quality of virtualization, which is the fundamental and enabling technology for cloud computing. Agent-based cloud computing is also introduced in [14], which means applying agent-based approaches to managing cloud computing infrastructures for high-performance computing.

\section{3) Mobile Technology}

One more leading cause of innovation for SMEs' business is the recently rising mobile technology. Mobiles devices are no longer just a consumer gadget. Tablets and smart-phones together with the Internet are quickly becoming indispensable tools in manufacturing environments.

Mobile devices due to their advantages of mobility, portability, availability and affordability, not only allow operators and managers to stay connected and completely in control of what happens in the plant at any time and in any place, enable efficient communication, but also allow to access information faster from anywhere in the world [15].

The development of the above three technologies and their integrations are driving a revolution in manufacturing and a shift in the way SMEs do businesses. With the aid of the Internet technology, they enable data and information share among different roles and stakeholders in real time and facilitate the popularization of e-manufacturing and ecommerce. Meanwhile, the advent of computer networks and information technology has also provided promising opportunities to upgrade manufacturing systems with intelligent control [16]. An integration of machines and manufacturing processes with real-time monitoring and remote control through computer networks has become imperative.

\section{4) Remote Monitoring}

A remote monitoring system is usually network-based and combined with other IT tools, e.g. Bluetooth and mobile devices. Based on a server/client-type computer network [17], software tools are loaded to client workstations and servers for 
remote monitoring and control. The service providers employ the tools to effectively handle their clients' requirements.

Remote monitoring (RM) systems can be designed and implemented to perform varied tasks, e.g., on-line monitoring of automated machine tools [17,18], detecting and reporting problems, remote diagnostics and maintenance of machines, enterprise internal production management and supply chain management [19]. Together with relevant IT management tools, the adoption of remote monitoring technology can help to automate manufacturing processes and greatly improve flexibility, responsiveness, and productivity, for that technicians are able to monitoring multiple client workstations simultaneously and perform easier maintenance tasks. Via remote monitoring, the machine equipment can also be linked directly to the Internet-based e-manufacturing.

Other than mobile technology, agent technology and cloud services can both be integrated with remote monitoring systems, allowing direct control and data/service transmission over applications operating on the system and thus enhancing the overall system performance.

\section{B. Artificial Intelligence Techniques}

The most significant feature of IMS is that it utilizes artificial intelligence (AI) techniques for manufacturing activities. The intelligence system performs manufacturing functions as if human does. Computer as a current essential device, can not only perform the respective functions but also make information systems capable of integrating manufacturing functions.

\section{1) Computer-Aided Systems}

Tools such as systems of computer-aid design (CAD), computer-aided manufacturing (CAM), computer-aided engineering (CAE), computer-integrated manufacturing (CIM), computer-aided process planning (CAPP) and computer-aided quality control (CAQC) have been constantly developed and improved. Effective utilization of computer systems in manufacturing has improved efficiency of manufacturing through increasing productivity and quality as well as flexibility and decreasing lead times, work in process inventories and costs, and thus brought huge benefits [20].

\section{2) Numerical Control Machines and Robots}

One of the most widely known AI techniques is probably robotics. Increasing applications of mobile robots and numerical controlled (NC) machine tools with computers as an integral part of their control, highly supports the development of intelligent manufacturing systems with high flexibility and autonomy and reduces the workload of human labors. In modern CNC systems, component design is highly automated using CAD/CAM systems. Dimensional errors and lack of standardized performance evaluation procedure as some potential problems that might occur when adopting robotic machining technology, discussed in [21].

To make computer systems more intelligently requires advanced computing technology. A lot of methodologies have been developed to achieve artificial intelligence, such as expert systems, artificial neural networks, genetic algorithms, fuzz logic, graph theory, holon philosophy, intelligent agents, just to name a few $[20,22]$.

\section{3) Virtual Reality}

Virtual reality (VR) is a novel digital technology which is often regarded as a natural extension to 3D computer graphics with advanced input and output devices [23]. VR technology allows a user to step through the computer screen into a simulated three-dimensional (3D) world. An artificial environment is created in which human user can look at, move around, and interact with the environment as if it is real.

One of the most highlighted applications is that virtual reality can assist with the integration of 3D design and manufacturing tools, e.g. facilitating the design of a product, allowing 3D assembly evaluation. The integration of VR technology with software systems for engineering, design, and manufacturing will boost to the field of computer-aided engineering (CAE) [23]. More applications of virtual reality in manufacturing areas and benefits from the applications are discussed in [24]. Concepts of virtual prototyping (VP), virtual assembly (VA), and virtual manufacturing (VM) are also introduced in $[23,25]$. A virtual prototyping system that integrates virtual reality with rapid prototyping (RP) to create virtual or digital prototypes to facilitate product development is described in [25].

VR can be a powerful tool for testing and evaluating new products and ideas before being employed in practical manufacturing, preventing costly mistakes, decreasing time to market, and meanwhile increasing worker safety. However, widespread use and acceptance of virtual reality will require devices and software with higher quality and lower cost.

\section{Others}

\section{1) Rapid Prototyping and Manufacturing}

Additive manufacturing (AM) is defined as the "process of joining materials to make objects from 3D model data, usually layer upon layer" [26,27]. AM technology opens up the possibility of rapid prototyping, rapid tooling and rapid manufacturing. Relevant techniques include 3D printing, stereolithography apparatus (SLA), selective laser sintering (SLS), fused deposition modeling (FDM), laminated object manufacturing (LOM), ballistic particle manufacturing (BPM), solid ground curing (SGC), etc. Based on the principle of additive machining, these AM techniques make it possible to construct components with complex geometries by means that are not easy to produce using conventional removal machining methods.

Rapid prototyping and manufacturing (RP\&M) building on AM techniques have shown a high potential to substantially reduce the cycle time and cost of product development by quickly creating 3D prototypes or products. In addition, RP\&M significantly improve the flexibility for manufacturing small batch size products and a variety of types of products and also increase a product's functionality $[27,28]$. Furthermore, due to the capability of AM to realize optimized design and construct complex geometries, RP\&M enables environmental friendly product design and manufacturing through eliminating waste and reducing the amount of energy, 
fuel, or natural resources required for operation [27]. Application areas of RP\&M can be found in [28].

\section{2) 3D Measurement and Inspection}

Precision inspection has been widely used in manufacturing to measure the dimensional accuracy of parts and products in order to meet the quality requirements, especially for parts/products with free-form and complex surfaces [29]. A useful tool in the areas of performing 3D measurement and inspection is $3 \mathrm{D}$ scanner. The analytical technology of 3D laser scanning can also be applied to RP\&M and reverse engineering, in addition to production-related quality control.

A 3D scanner can be integrated into an automated measuring cell (e.g. controlled by robots) in order to perform automated measurement and inspection, which is mainly available for small to medium parts and components. Removing/reducing manual operations from the measurement process and being able to measure parts repeatedly and quickly undoubtedly help to increase accuracy and efficiency $[30,31,32]$.

\section{3) Reverse Engineering}

Reverse engineering (RE) is a process of measuring, analyzing, and testing to reconstruct the mirror image of an object or retrieve a past event [33]. RE is practiced by manufacturers primarily in support of design and product development, bringing substantial business benefits in shortening the product development cycle, reducing the production period and costs. Starting with a finished product or an existing part without available design data, engineers work backward to discover the underlying techniques and reproduce the original model, often digitized in a CAD environment where modifications can be made for improvement [33,34].

RE is a highly knowledge-based technology of reinvention and needs highly skilled engineers and technicians. This manner is primarily adopted by large companies in the mature industries of aviation and automobile. Today more and more emerging technologies (e.g. 3D scanning) and the extensive use of $\mathrm{CAD} / \mathrm{CAM} / \mathrm{CAE}$ systems are making reverse engineering easier and more accurate. RE is becoming widely used in the application areas such as mechanical industry, consumer electronics industry, software and IT industries, medical device industries, etc. [34]

Reverse engineering should integrate the legal, ethical, economic, environmental, and other realistic constraints into consideration. Adequate legal acceptance of RE is crucial for further discovering technical and social benefits [34].

\section{4) Reconfigurable Machine Tools}

A reconfigurable manufacturing system (RMS) is introduced in [3,5], with comparison to dedicated manufacturing lines and flexible manufacturing systems. CNC machines and reconfigurable machine tools (RMT) are the main components of RMS [3]. RMT is a modular machine with a changeable structure that allows adjustment of its resources, and eventually allows changes in customer demand volume and variety of products. RMT is economical for medium-volume production, highly variant in production line, and can fill the gap between dedicated machine tools and CNC techniques. To make RMT possible to use, enablers like interface standards, comprehensive software and an openarchitecture controller system are needed.

\section{APPLICATIONS}

The application domains of all the diverse methods and technologies into manufacturing can be varied, e.g., engineering design, prototyping, material handling, process planning, production planning, scheduling and resource allocation, process control, monitoring and diagnosis, assembly, enterprise integration, execution control, quality and operations management, supply chain management, lifecycle management, etc. These are combined into four major areas: (1) design, (2) planning, (3) production, and (4) system level activities [22]. A hierarchical framework of detailed manufacturing functions at four levels can be found in [20]. In this paper, a circular framework of detailed manufacturing activities is proposed for SIMS. The whole framework can be divided into five groups:

1) Core: The elements of human, facilities including computer and networks, information and communication makes up the core part of SIMS.

2) Inner circle: The activities in this circle (grey) are mainly from the perspective of material flow, from procurement logistics (e.g. raw materials procurement from the supplier market and delivery), material requirement planning (or manufacturing resource planning), to material handling and processing. Internal manufacturing logistics in the manufacturing process and inventory storage, both in terms of materials and products are also included in this circle.

3) Outer circle: This circle (white) is from the generalized perspective of products, from product design according to customer demands and feedback, product prototype testing and optimization, process planning, manufacturing process (including part production, inspection, and assembly), to distribution logistics (e.g. finished product sales and distribution to the customer market).

4) Arrowed cycle: The outer cycle with arrows supplements corresponding control and management activities at the system level, such as material flow control, production activity control, quality control, inventory control, their supporting management systems (e.g. total quality management and continuous improvement), logistics and supply chain management, and other business activities in the life cycle (e.g. capacity/demand management, information/ knowledge management, operations/waste management, sales and marketing, enterprise integration and collaboration, etc).

5) Outermost: The aforementioned tools and technologies are listed according to their application areas.

In order to fully utilize these new technologies in IMS, several technologies are often integrated together leading to the emerging of diverse concepts and new paradigms. The integration of advanced technologies such as CAD/CAM, 
CNC, robots, and automated material handling systems, forms a computer-integrated manufacturing (CIM) system where computer plays an integral role. Today computers are also extensively involved in business management activities such as enterprise resource planning, customer satisfaction evaluation, and quality management and improvement.

A new manufacturing paradigm called direct digital manufacturing (DDM) is introduced in [35], with comparison to craft production, mass production and mass customization. Different from CIM, the development of DDM is greatly attributed to both the RP\&M technology and the technological breakthrough of computer and information technology. As defined in [35], DDM is an interconnection of additive manufacturing equipment, computers through a network (e.g.
Internet and servers) and computer software. The integrating systems of CAD/CAM and RP\&M highly improve the capability of rapid product development.

The engineering design is mainly supported by $3 \mathrm{D}$ CAD modeling using parametric modeling techniques, CAE for simulation and analysis, and CAM for NC simulation, allowing the optimization of product manufacturing [34,36]. The virtual techniques and rapid prototyping technologies facilitate the validation of design concept via simulation and prototyping. Automation is the primary advantage of implementing industrial robots and CNC machine tools, which subsequently leads to other benefits, such as speedy production, cost savings, easy operation, and free manufacturing of complex geometric design [33].

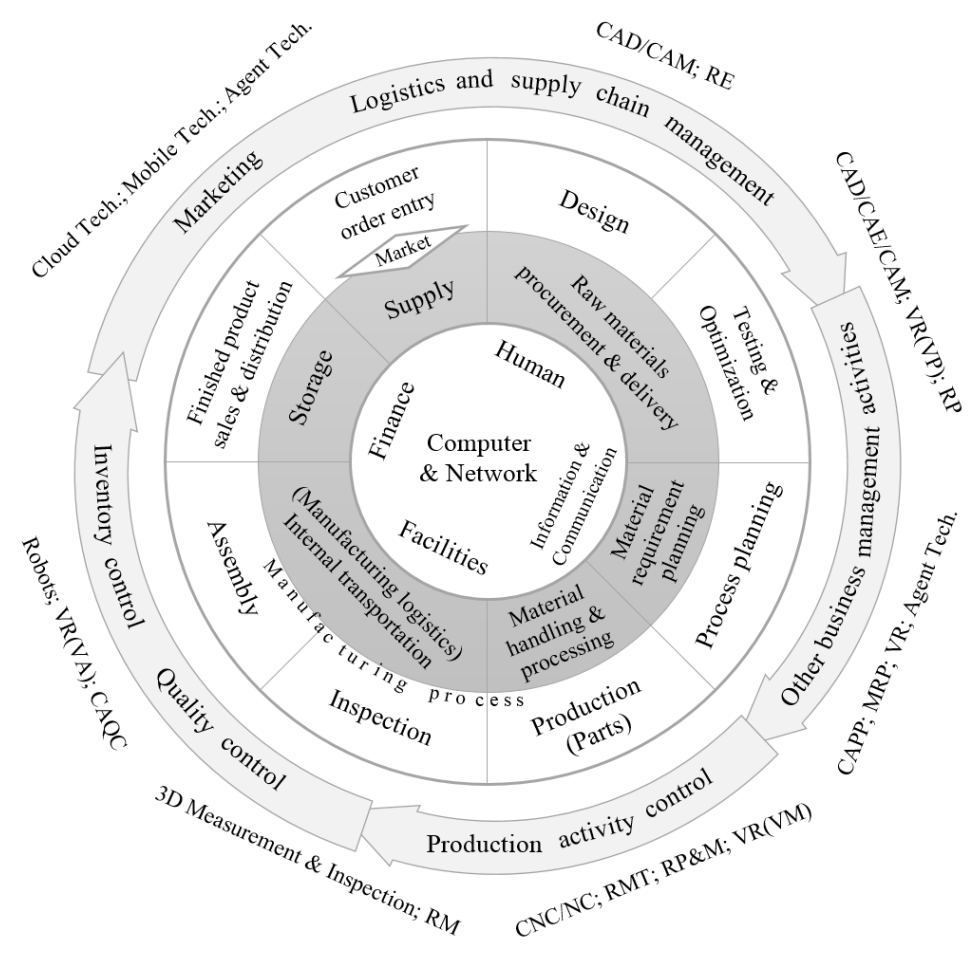

Fig. 2. Activity components of small-scale intelligent manufacturing system and applications of supporting tools and technologies.

While stimulated and enabled by computers and modern digital and automation technology, both DDM and CIM support low-medium volume production and make the manufacturing industry capable to provide rapid response to the changing needs of customers. SMEs rely on these digital approaches and advanced manufacturing technologies and the integration of design with construction and production through the use of 3D modeling software and additive or subtractive manufacturing processes. Meanwhile, the advent of computer networks and information and communication technology has offered promising opportunities to upgrade manufacturing systems with intelligent controls and change business processes and management styles.
Due to the rapid development of IT, another emerging paradigm named Industry 4.0 is on the way to bring new industrial revolution. In such a global manufacturing environment, the system is vertically networked with business processes within enterprises and horizontally connected to disperse partner networks across the entire value chain that can be managed in real time [37]. The Cyber-Physical Systems (CPS) comprise smart machines, storage systems and production facilities capable of autonomous actions and control, while the Internet of Things (IoT) significantly adds intelligence to communication networks [38]. Other enabling technologies such as mobile technology, cloud computing, big data analytics, virtual reality, additive manufacturing or 3D printing, and industrial robots can be drawn together to increase productivity, 
quality, flexibility and agility within the manufacturing industry and facilitate global manufacturing via the Internet $[25,39]$.

All these new and promising paradigms contribute to the shaping of SIMS. Manufacturing businesses nowadays become increasingly IT-reliant, globalized, distributed and agiledemanding [12]. In order to survive in this rapidly changing manufacturing environment, SMEs should keep up with these trends and have to be more and more competitive across the entire range of business functions such as marketing and sales, not merely within product design and manufacturing.

\section{CONCLUSIONS}

Facing intense competition in the global market, the manufacturing industry is forced to compete effectively by reducing time to market and cost while assuring high quality products preferably with desired features. Agility has become the key to survival and success in businesses and strengthening customer relationships. Meanwhile, advances in technology have blurred the boundaries between the physical and digital worlds. Under this Industry 4.0 environment, SMEs are also driven to actively seek innovative solutions in order to attain the competitive edge.

The novel concept of small-scale intelligent manufacturing system was put forward in this paper. Several pertinent and promising manufacturing techniques and enabling technologies were discussed. Each of them has particular features and might suit different types of manufacturing enterprises. Further study can focus on developing an approach to select the most suitable and cost-effective tools in the purpose of providing suggestions to SMEs. Some present problems and research issues on each technology were also mentioned, indicating that the future development of SIMS relies on the research and development (R\&D) of these technologies to maximize their potential capacities on time and cost savings.

As a result of applications, SIMS will be highly integrated, automated and flexible, and allow timely response to increasing customer demands for quick delivery of orders thus to achieve the highlighted property of agility. Moreover, SIMS will become more networked, digital, and knowledge-based. It is expected that SIMS will bring new opportunities and advantages to fulfill the potential of SMEs in creating economic, social, and environmental impact.

\section{ACKNOWLEDGMENT}

This research was carried out with the support from TARGET project financed by EU Northern Periphery and Arctic (NPA) Programme, and the project aims at improving the competitiveness of the manufacturers in NPA region.

\section{REFERENCES}

[1] P. Rodriguez, A. Dasgupta, A. Patwardhan, H.K. Mittal, S. Nagpal, T. Karunakaran, and N. Saxena, Report of the Working Group on Science \& Technology for Small \& Medium Scale Enterprises (SMEs) for the Eleventh Five Year Plan (2007-2012), 2012. Accessed 08 September 2015, <http://www.dst.gov.in/sites/default/files/rep-subsme.pdf>.

[2] A. Isaksen, and K. Smith, Innovation Policies for SME's in Norway: Analytical Framework and Policy Options. Oslo: STEP Group, 1997.
[3] Y. Koren, U. Heisel, F. Jovane, T. Moriwaki, G. Pritschow, G. Ulsoy, and H.V. Brussel, "Reconfigurable manufacturing systems," Annals of the CIRP, vol. 48, pp. 527-540, 1999.

[4] NIST, Programs of the Manufacturing Engineering Laboratory, 2010. Accessed $19 \quad$ August 2015 , <http://www.nist.gov/el/upload/melprograms10.pdf>.

[5] M.G. Mehrabi, A.G. Ulsoy, and Y. Koren, " Reconfigurable manufacturing systems: key to future manufacturing," Journal of Intelligent Manufacturing, vol. 11, pp. 403-419, 2000.

[6] H.P. Wiendahl, H.A. ElMaraghy, P. Nyhuis, M.F. Zäh, H.H. Wiendahl, N. Duffie, and M. Brieke, "Changeable manufacturing - classification, design and operation,” Annals of the CIRP, vol. 56, pp. 783-809, 2007.

[7] L. Monostori, J. Váncza, and S.R.T. Kumara, “Agent-based systems for Manufacturing,” Annals of the CIRP, vol. 55, pp. 697-720, 2006.

[8] P. Leitão, “Agent-based distributed manufacturing control: a state-ofthe-art survey," Engineering Applications of Artificial Intelligence, vol. 22, pp. 979-991, 2009.

[9] Q. Guo, and M. Zhang, “A novel approach for multi-agent-based intelligent manufacturing system,” Information Sciences, vol. 179, pp. 3079-3090, 2009.

[10] W. Shen, and D.H. Norrie, “Agent-based systems for intelligent manufacturing: a state-of-the-art survey," Knowledge and Information Systems, vol. 1, pp. 129-156, 1999.

[11] W. Shen, Q. Hao, H.J. Yoon, and D.H. Norrie, “Applications of agentbased systems in intelligent manufacturing: an updated review, " Advanced Engineering Informatics, vol. 20, pp. 415-431, 2006.

[12] X. Xu, "From cloud computing to cloud manufacturing," Robotics and Computer-Integrated Manufacturing, vol. 28, pp. 75-86, 2012.

[13] A. Shawish, and M. Salama, "Cloud computing: paradigms and technologies,” in Inter-cooperative Collective Intelligence: Techniques and Applications, F. Xhafa and N. Bessis, Eds. Berlin: Springer, 2014, pp. 39-67.

[14] K.M. Sim, “Agent-based cloud computing,” IEEE Transactions On Services Computing, vol. 5, no. 4, pp. 564-577, 2012.

[15] http://www.automationworld.com/3-reasons-use-mobile-technologymanufacturing

[16] T.H. Hou, W.L. Liu, and L. Lin, “Intelligent remote monitoring and diagnosis of manufacturing processes using an integrated approach of neural networks and rough sets," Journal of Intelligent Manufacturing, vol. 14, pp. 239-253, 2003.

[17] M.C. Pan, P.C. Li, and Y.R. Cheng, "Remote online machine condition monitoring system,” Measurement, vol. 41, pp. 912-921, 2008.

[18] L. Wang, P. Orban, A. Cunningham, and S. Lang, "Remote real-time CNC machining for web-based manufacturing," Robotics and CompterIntegrated Manufacturing, vol. 20, pp. 563-571, 2004.

[19] S. Zhou, W. Ling, and Z. Peng, “An RFID-based remote monitoring system for enterprise internal production management," International Journal of Advanced Manufacturing Technology, vol. 33, pp. 837-844, 2007.

[20] L. Benyoudef, and B. Grabot, Artificial Intelligence Techniques for Networked Manufacturing Enterprises Management. Paris: Springer, 2010.

[21] J.D. Barnfather, M.J. Goodfellow, and T. Abram, “A performance evaluation methodology for robotic machine tools used in large volume manufacturing," Robotics and Computer-Integrated Manufacturing, vol. 37, pp. 49-56, February 2016.

[22] R. Teti, and S.R.T. Kumara, "Intelligent computing methods for manufacturing systems," Annals of the CIRP, vol. 46, pp. 629-652, 1997.

[23] S. Jayaram, H.I. Connacher, and K.W. Lyons, "Virtual assembly using virtual reality techniques," Computer-Aided Design, vol. 29, no. 8, pp. 575-584, 1997.

[24] T.S. Mujber, T. Szecsi, and M.S.J. Hashmi, “Virtual reality applications in manufacturing process simulation," Journal of Materials Processing Technology, vol. 155-156, pp. 1834-1838, 2004.

[25] S.H. Choi, A.M.M. Chan, "A virtual prototyping system for rapid product development," Computer-Aided Design, vol. 36, pp. 401-412, 2004. 
This copy is the accepted manuscript by SIMS 2016.

The published version is available on IEEE Xplore: http://ieeexplore.ieee.org/document/7802896/

[26] ASTM, Standard Terminology for Additive Manufacturing Technologies, 2010. Accessed 31 January 2016, <http://enterprise.astm.org/filtrexx40.cgi?+REDLINE_PAGES/F2792.ht $\mathrm{m}>$

[27] S.H. Huang, P. Liu, A. Mokasdar, and L. Hou, “ “Additive manufacturing and its societal impact: a literature review," International Journal of Advanced Manufacturing Technology, vol. 67, pp. 1191-1203, 2013.

[28] X. Yan, and P. Gu, “A review of rapid prototyping technologies and systems," Computer-Aided Design, vol. 28, no. 4, pp. 307-318, 1996.

[29] Y. Li, P. Gu, "Free-form surface inspection techniques state of the art review,” Computer-Aided Design, vol. 36, pp. 1395-1417, 2004.

[30] http://www.t3dmc.com/automation/

[31] http://www.atos-core.com/en/applications.php

[32] http://www.gom.com/news/latest-news/atos-scanbox-4105.html

[33] W. Wang, Reverse Engineering: Technology of Reinvention. Abingdon: Taylor and Francis Group, 2010, pp. 1-23.

[34] A. Kumar, P.K. Jain, and P.M. Pathak, "Reverse engineering in product manufacturing: an overview," Chapter 39 in DAAAM International Scientific Book, B. Katalinic and Z. Tekic, Eds. Vienna: DAAAM International, 2013, pp. 665-678.
[35] D. Chen, S. Heyer, S. Ibbotson, K. Salonitis, J.G. Steingrímsson, and S. Thiede, "Direct digital manufacturing: definition, evolution, and sustainability implications," Journal of Cleaner Production, vol. 107, pp. 615-625, 2015.

[36] H.G. Lemu, "Virtual engineering in design and manufacturing," Advances in Manufacturing, vol. 2, pp. 289-294, 2014.

[37] M. Hermann, T. Pentek, and B. Otto, “Design Principles for Industrie 4.0 Scenarios, ” 2015. Accessed 31 January 2016, <http://www.snom.mb.tudortmund.de/cms/de/forschung/Arbeitsberichte/Design-Principles-forIndustrie-4_0-Scenarios.pdf>.

[38] A. Arsénio, H. Serra, R. Francisco, F. Nabais, J. Andrade, and E. Serrano, "Internet of intelligent things: bringing artificial intelligence into things and communication networks, " in Inter-cooperative Collective Intelligence: Techniques and Applications, F. Xhafa and N. Bessis, Eds. Berlin: Springer, 2014, pp. 1-37.

[39] http://www.zdnet.com/article/industry-4-0-its-all-about-informationtechnology/

[40] N. Cross, Engineering Design Methods: Strategies for Product Design, 4th ed. New Jersey: Wiley, 2008. 